\title{
BMJ Open Developing an ecological framework of factors associated with substance use and related harms among Aboriginal and Torres Strait Islander people: protocol for a systematic review
}

\author{
Mieke Snijder, ${ }^{\oplus 1}$ Briana Lees, ${ }^{\oplus 1}$ James Ward, ${ }^{2,3}$ Annalee E Stearne, ${ }^{4}$ \\ Nicola Clare Newton, ${ }^{1}$ Lexine Stapinski ${ }^{1}$
}

To cite: Snijder M, Lees B, Ward J, et al. Developing an ecological framework of factors associated with substance use and related harms among Aboriginal and Torres Strait Islander people: protocol for a systematic review. BMJ Open 2019;9:e024418. doi:10.1136/ bmjopen-2018-024418

- Prepublication history and additional material for this paper are available online. To view these files, please visit the journal online (http://dx.doi org/10.1136/bmjopen-2018024418).

Received 25 May 2018 Revised 26 February 2019 Accepted 27 February 2019

Check for updates

(c) Author(s) (or their employer(s)) 2019. Re-use permitted under CC BY-NC. No commercial re-use. See rights and permissions. Published by BMJ.

For numbered affiliations see end of article.

Correspondence to

Dr Mieke Snijder;

mieke.snijder@sydney.edu.au

\section{ABSTRACT}

Introduction Aboriginal and Torres Strait Islander people experience high rates of substance use and related harms. Previous prevention programmes and policies have met with limited success, particularly among youth, and this may be a result of inadequately targeting the unique risk and protective factors associated with substance use for Aboriginal and Torres Strait Islander people. The purpose of this systematic review is to therefore synthesise the risk and protective factors associated with substance use and related harms among Aboriginal and Torres Strait Islander people, and critically appraise the methodological quality of the included studies.

Methods and analysis A total of seven peer-reviewed (Cochrane, Embase, Psychlnfo, Medline, ProQuest, Informit, and CINAHL) and two grey literature (Heath/nfoNet and Closing the Gap Clearinghouse) databases will be systematically searched using search terms in line with the aims of this review and based on previous relevant reviews. Studies published between 1 January 1990 and 31 April 2018 will be included if they identify risk and/or protective factors for substance use or related harms in a study sample that consists of at least $50 \%$ Aboriginal and Torres Strait Islander people. A narrative synthesis will be undertaken where the identified factors will be organised using an ecological approach into individual, relationship, community, societal and cultural levels. A critical appraisal of study quality will be conducted using the Joanna Briggs Institute Critical Appraisal Checklist for Studies Reporting Prevalence Data and the qualitative assessment tool by Godfrey and Long.

Ethics and dissemination Formal ethics approval is not required as primary data will not be collected. The results will be disseminated through a peer-reviewed publication, conference presentations and social media.

PROSPERO registration number CRD42017073734.

\section{INTRODUCTION}

Aboriginal and Torres Strait Islander people have the oldest continuing culture in the world. ${ }^{1}$ Like other Indigenous Peoples around the world, many Aboriginal and Torres Strait
Strengths and limitations of this study

- This systematic review will be the first to synthesise the risk and protective factors associated with substance use and related harms among Aboriginal and Torres Strait Islander people, identified from peer-reviewed and grey literature publications.

- This review will move away from an individual-level focus on substance use prevention towards an ecological approach, which can be used by programme developers, researchers and policy-makers when deciding on intervention strategies.

- The expected heterogeneous outcomes of the included papers means we will not conduct a meta-analysis and will likely reduce the quality of the final model.

Islander people's livelihoods were severely disrupted on colonisation, with people often forcibly removed from their traditional lands and prevented from utilising their traditional laws and culture. This has resulted in negative impacts on well-being and sense of place in contemporary society, including a reduced sense of self-determination. ${ }^{2}$ The cycle of dispossession and disempowerment resulting from colonisation, combined with ongoing policy failures in social services and health, continues to negatively impact many Aboriginal and Torres Strait Islander people, as evidenced by the poorer health and social outcomes when compared with non-Indigenous Australians. ${ }^{3-9}$

Despite evidence of resilience in dealing with poorer outcomes on social determinants of health, Aboriginal and Torres Strait Islander people experience high rates of substance use and related harms. ${ }^{3}{ }^{10}$ For example, among Aboriginal and Torres Strait Islander people aged 44 years and younger, 
mental health and substance use disorders are the leading cause of disease and they account for $14 \%$ of the health gap between Aboriginal and Torres Strait Islander people and non-Indigenous Australians. ${ }^{11}$ As substance use is a modifiable risk factor for disease, it is therefore an important target area to close the gap in health outcomes between Aboriginal and Torres Strait Islander people and non-Indigenous Australians. ${ }^{12} 13$

While past prevention programmes and policies have made some improvements in substance use-related outcomes over the past decades, these achievements have been slow and insufficient to reduce substance use and related harms among Aboriginal and Torres Strait Islander people. ${ }^{14-17}$ For example, a recent literature review found that only three out of the six substance use prevention programmes for Aboriginal and Torres Strait Islander adolescents were effective in improving substance-related outcomes. ${ }^{18}$ One explanation for the reduced impact of policies and programmes could be that they do not adequately target the unique risk and protective factors that are associated with substance use for Aboriginal and Torres Strait Islander people. Identifying these specific factors has the potential to improve policies and programmes that aim to reduce substance use and related harms experienced by Aboriginal and Torres Strait Islander people.

In assessing factors associated with substance use and related harms, it is beneficial to take an ecological approach to ensure that a comprehensive overview is created of the various factors influencing the outcomes of interest. ${ }^{19} 20$ In addition to influences of colonisation, intergenerational trauma and disempowerment, substance use and related harms are influenced by a variety of individual, community, relationship, societal and cultural factors. ${ }^{20-22}$ While previous reviews have investigated the association of these factors with substance use for mainstream populations, ${ }^{19}{ }^{23-26}$ to date, no study has synthesised the evidence of risk and protective factors on all ecological levels associated with substance use and related harms for Aboriginal and Torres Strait Islander people in Australia. It is therefore the purpose of this literature review to provide an overview of the risk and protective factors associated with substance-related outcomes, organised by ecological levels. By focusing on Aboriginal and Torres Strait Islander populations in Australia, this will provide the most robust evidence base to inform the development of culturally appropriate substance use prevention programmes for Aboriginal and Torres Strait Islander people, to ultimately reduce the health gap between Indigenous and non-Indigenous Australians.

\section{METHODS AND ANALYSIS}

This protocol follows the Preferred Reporting Items for Systematic Reviews and Meta-Analysis Protocols (PRISMA-P) statement, ${ }^{27}{ }^{28}$ found in the online supplementary file. This protocol has been registered in the
Table 1 Search strategy for systematic review of risk and protective factors of substance use and related harms among Aboriginal and Torres Strait Islander people (eg, Medline search)

1 ((Aborigin* OR 'Torres Strait Islander' OR Indigenous) and Australia).mp.

2 ((risk OR protec* OR resilienc* OR underlying OR ecological OR vulnerab* OR psychosocial) AND (factor OR mechanism OR character*).mp.

3 (wellbeing OR mental health OR substance OR alcohol OR drug OR tobacco).mp.

4 limit 1 to $y r=' 1990-2017$ '

*mp=title, abstract, original title, name of substance word, subject heading word, keyword heading word, protocol concept word, rare disease concept word, unique identifier, synonyms.

PROSPERO registry of the University of York (registration number: CRD42017073734).

\section{Objectives}

The overall objective of this review is to identify and synthesise the risk and protective factors associated with substance use and related harms among Aboriginal and Torres Strait Islander people. Specifically, this review will aim to answer the following two questions: (1) What are the risk and protective factors for substance use and related harms among Aboriginal and Torres Strait Islander people, across individual, relationship, community, cultural and societal levels? and (2) What is the methodological quality of studies assessing the factors related to substance use and related harms for Aboriginal and Torres Strait Islander people?

\section{Search strategy}

The relevant literature published between 1 January 1990 and 30 April 2018 will be systematically searched in seven electronic peer-reviewed databases and two grey literature databases for quantitative and qualitative studies. Peer-reviewed databases will include: Cochrane, Embase, PsychInfo, Medline, ProQuest, Informit and CINAHL. Following previous literature reviews relating to Aboriginal and Torres Strait Islander health, ${ }^{29}{ }^{30}$ the grey literature databases will be: HeathInfoNet and Closing the Gap Clearinghouse. Table 1 provides an example of the search strategy that will be used in Medline, which will be replicated for the other electronic databases. The reference lists of selected studies will be assessed for further relevant publications and papers will be solicited from researchers in the field.

Search terms will be used to identify studies assessing the risk and protective factors of substance use and related harms for Aboriginal and Torres Strait Islander people. The search terms will be based on previous reviews into factors associated with substance use in adolescents in mainstream populations ${ }^{19}$ and factors associated with wellness for Indigenous Peoples. ${ }^{20}$ Search terms will be combinations of keywords related to the participants 
('Aborigin* OR 'Torres Strait*' OR Indigenous' AND Australia), the outcomes (wellbeing OR 'mental health' OR substance OR alcohol OR drug OR tobacco) and the factors ('risk' OR protec* OR resilienc* OR underlying OR ecological OR vulnerab* OR 'psychosocial' AND 'factor OR mechanism OR character*'). Two reviewers will independently screen articles, extract data and assess the methodological quality.

\section{Eligibility criteria}

Eligibility criteria for this review are defined using PICOS (population, intervention/exposure, comparator, outcome, study characteristics) ${ }^{28}$ Inclusion criteria will be as follows: (1) $50 \%$ or more of the study sample are Aboriginal and Torres Strait Islander people, or a sub-analysis has been conducted for Aboriginal and Torres Strait Islander people when sample is less than $50 \%$; (2) the study identifies or measures risk and/or protective factors for substance use or related harms; (3) the outcome of interest is substance use or related harms; (4) the study was published between 1 January 1990 and 30 April 2018; (5) full-text version is available and (6) the publication includes a new data analysis. A more detailed description and definition of all PICOS criteria follows.

\section{Population}

Studies will be considered if they contain a sample where at least $50 \%$ of participants identified as Aboriginal and Torres Strait Islander people. Studies will also be considered if the participant sample included less than $50 \%$ of Aboriginal and Torres Strait Islander people but a separate analysis for Aboriginal and Torres Strait Islander people was provided. Participants of all ages will be included.

\section{Exposure}

Studies must identify and measure risk and protective factors for substance use and related harms. The factors will be organised using an ecological approach, which includes factors on individual, relationship, community, societal, and culturally distinct levels ${ }^{20}$ (see figure 1 for proposed model). Each ecological level is defined as follows:

- Individual-level factors include biological and personal history factors such as age, socioeconomic status, health and psychosocial factors.

- Relationship-level factors include close relationships with peers, partners and family members who influence an individual's behaviour.

- Community-level factors include the individual's lifestyle settings such as the workplace or geographical location in which social relationships occur.

- Societal-level factors include social norms, social policy and availability of substances.

- Culturally distinct-level factorsinclude historical contexts, languages, cultural practices, spirituality, values and social structures that are specific to cultural groups.

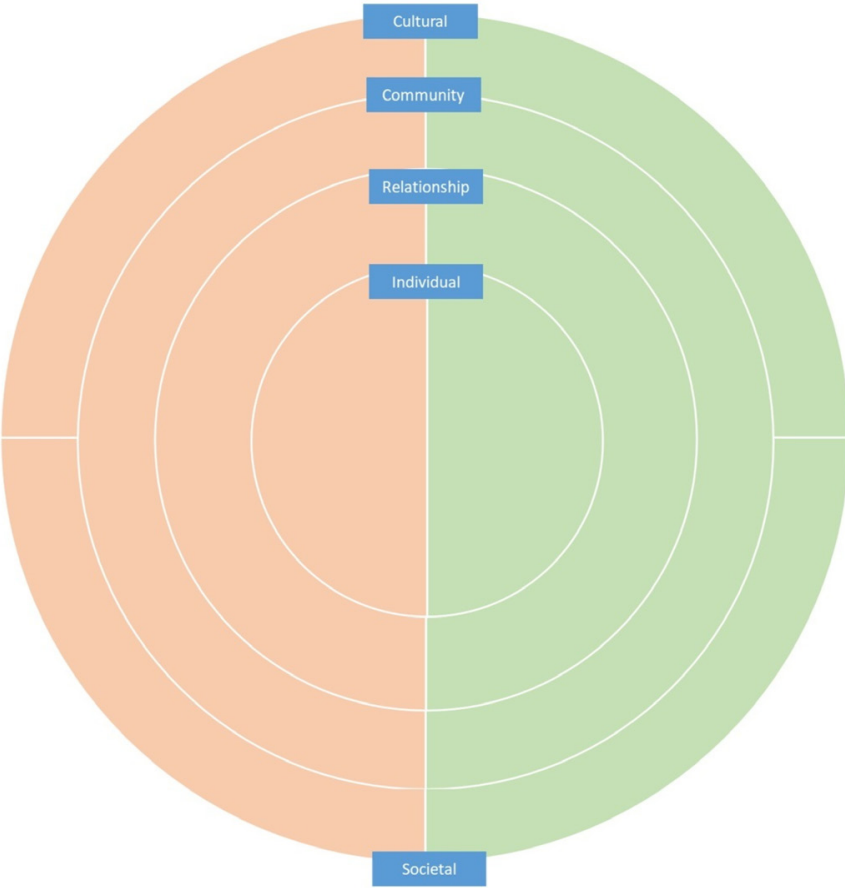

Figure 1 Proposed ecological model resulting from the systematic literature review.

\section{Comparator}

No comparator or control group is necessary for studies to be included in this review. Studies that do include a comparator must compare Aboriginal and Torres Strait Islander people who use substances (or are heavy users) with Aboriginal and Torres Strait Islander people who do not use substances (or are not heavy users). Papers comparing Aboriginal and Torres Strait Islander people and non-Indigenous Australians will not be included in this review.

\section{Outcomes}

The primary outcomes of interest are substance use and associated harms. Outcome types will be classified based on previous research ${ }^{19}$ which categorised substance use and related outcomes into three levels: (1) use or frequency; (2) regular, problem, heavy use or binge and (3) use disorder or abuse or dependence. Following Stone et al (2012), the wording of each category will be consistent across risk and protective factors for inclusion in tables, but categories are defined separately. For risk factors the following definitions will be used:

- Use or frequency refers to increased likelihood of substance use initiation, irregular substance use or frequency of use not further specified.

- Regular, problem, heavy use or binge. Regular use is an increase in use to at least weekly substance use. Heavy and binge use is an increase in large consumption during one occasion of use. Problem use is an increase in use that causes problems, including substance related injuries or hospitalisations. 
- Use disorder or abuse or dependence categorises increases in use and symptoms consistent with probable substance use disorder.

For protective factors the following definitions will be used:

- Use or frequency refers to a delay in initiation, an increase in abstinence or a reduction in frequency of use not further specified.

- Regular, problem, heavy use or binge refers to cessation or reduction of use following a period of regular, heavy or problem substance use and a reduction in substance-related harms.

- Use disorder or abuse or dependence refers to substance reduction or recovery from substance use disorder.

\section{Study characteristics}

Quantitative observational studies (cross-sectional, longitudinal, cohort and case-control studies) and qualitative studies that provide original data will be included. Reviews, information in books or letters will not be included.

\section{Selection procedure}

Reviewer one (BL) will screen all titles and abstracts from the peer-reviewed databases to determine eligibility for inclusion in the review. Reviewer two (MS) will independently screen a random selection of $25 \%$ of abstracts to ensure accuracy in the study selection. Reviewer one will screen and assess the eligibility of publications from the grey literature and reference lists of other identified papers. Full-text versions of the potentially eligible studies will be assessed by both reviewers to further determine eligibility for inclusion. Cohen's kappa will be calculated to assess the inter-rater agreement between the two reviewers at the full-text screening stage. Consultation between reviewers will be held at the time of abstract screening and full-text assessment to reconcile any differences of opinion. If there is no consensus, the senior author (LS) will assess the eligibility of the study.

An excel spreadsheet will be utilised to record the study title, authors, year, database and whether the study meets the eligibility criteria and should be included in the review. During abstract screening and full-text assessment, reasons for inclusion (PICOS) and exclusion will be recorded in the spreadsheet. Records from this spreadsheet will be used to generate the Preferred Reporting Items for Systematic Reviews and Meta-Analyses flowchart (see figure 2$).^{31}$

\section{Data management}

Bibliographic software (Endnote) will be used for the data management. All the search results from peer-reviewed databases, and screened publications from the grey literature, will be imported into the programme. Duplicates will be removed by reviewer one.

\section{Data extraction}

Data extraction will be completed by reviewer one, into an excel spreadsheet developed by the authors. The following elements will be extracted from each study: sample size and age, study population, percentage of

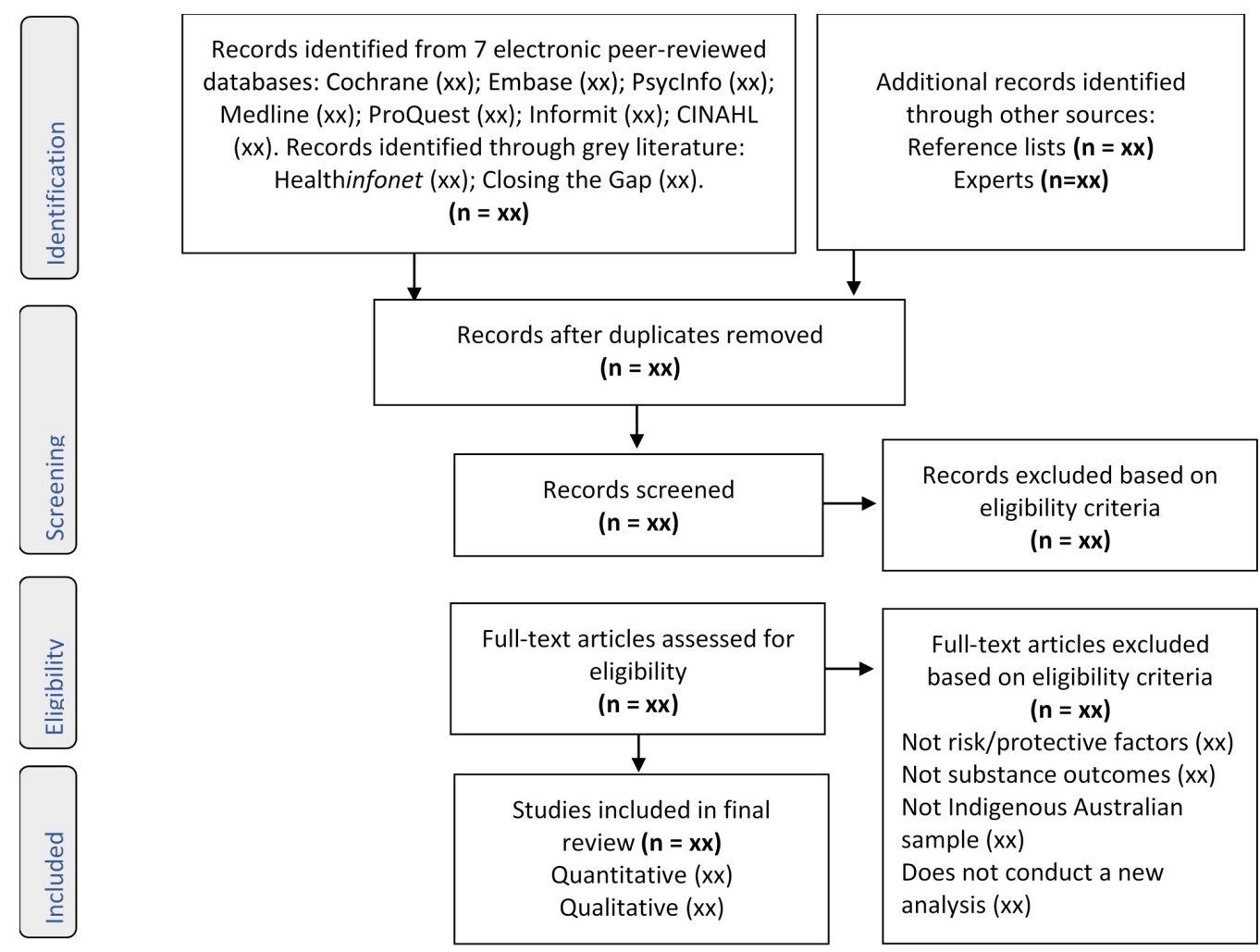

Figure 2 Preferred Reporting Items for Systematic Reviews and Meta-Analyses flow diagram of systematic review of studies assessing factors associated with substance use among Aboriginal and Torres Strait Islander people. 
Aboriginal and/or Torres Strait Islander descent, sex, study characteristics, substance-type measured and outcome type (use frequency, problem or heavy use, disorder or abuse), factors significantly associated with increased substance use and related harms (risk), factors significantly associated with reduced substance use and related harms (protective) and the effect size. To facilitate the comparison of risk and protective factors, odd ratios and/or rate ratios will be extracted from the studies. Where odds and/or rate ratios are not provided in the studies, we will calculate the odds ratios by extracting the total number of participants reported in each group of users or non-users who had the risk/protective factor or did not have the risk/protective factor. Where authors do not provide this information, we will contact the authors to ask them for these data.

Following previous reviews, ${ }^{19} 20$ a narrative synthesis of the main results extracted from the studies will be completed. A meta-analysis is likely not warranted given the heterogeneous outcome measures to be included in this review and the known variable quality of studies with Indigenous populations. ${ }^{32}$ A summary of each risk and protective factor statistically significantly associated with substance use and related harms will be reported in text, including effect sizes of specific studies. A table of risk and protective factor results will be produced, summarising the authors, study population, sample size and age, substance, outcome type and findings. The ecological framework will be utilised to organise the risk and protective factors within the table, grouping by individual, relationship, community, societal and culturally distinct levels. Findings will be discussed separately for each ecological level.

\section{Data analysis and quality assessment}

Following data extraction, the methodological quality of the studies will be assessed. The quality of quantitative studies will be appraised using the Joanna Briggs Institute Critical Appraisal Checklist for Studies Reporting Prevalence Data. ${ }^{33}$ This nine-question tool assesses quality across six domains: selection bias and sample size, study design, potentially confounding factors, data collection methods, statistical analysis and response rate. A scoring system of one-point per question will be used to determine the overall summary rating and allow comparability across publications. The quality of qualitative studies will be appraised using the qualitative assessment tool by Long et al. ${ }^{34}$ This will allow the reviewers to assess quality in four domains: sample, data collection, analysis and potential researcher bias, and policy and practical implications. A critical appraisal of all included studies will be completed by reviewer one and reviewer two will assess the quality of a random selection of $25 \%$ of included studies to ensure scoring accuracy. Consultation between reviewers will be held to reconcile any differences of opinion.

\section{Patient and public involvement}

Patients and the public were not involved in this systematic review protocol.

\section{Ethics and dissemination}

Ethical approval is not required for this study. The systematic review will be published in a peer-reviewed journal, presented at conferences and will be shared on social media platforms.

\section{CONCLUSION}

Aboriginal and Torres Strait Islander people experience a higher burden of disease from substance use and related harms compared with their non-Indigenous counterparts. ${ }^{11}$ Therefore, identifying which factors are associated with increased rates of substance use and related harms for Aboriginal and Torres Strait Islander people is critical to inform prevention programmes and policies. Identifying risk and protective factors associated with substance use will provide a framework that can improve understanding of substance use among Aboriginal and Torres Strait Islander people. This review will provide such a framework based on an ecological model which identifies factors on individual, relationship, community, societal and cultural levels that can lead to a reduction (protective) or increase (risk) in substance use and related harms.

By synthesising evidence regarding the risk and protective factors, this review will provide researchers, policy-makers and programme developers with robust evidence base to guide the development of prevention programmes and policies to specifically target the factors that are relevant for Aboriginal and Torres Strait Islander people. The review will also provide information about community and societal factors, and thus has the potential to guide government policies at a national and state level. This review seeks to improve health and social outcomes for Aboriginal and Torres Strait Islander people, by guiding the focus and tailoring of programmes and policies to lead to more effective and impactful substance use prevention initiatives.

\section{Author affiliations}

${ }^{1}$ The Matilda Centre for Research in Mental Health and Substance Use, University of Sydney, Sydney, Australia

${ }^{2}$ Infection and Immunity Theme, South Australia Health and Medical Research Centre, Adelaide, South Australia, Australia

${ }^{3}$ Faculty of Medicine Nursing and Health Sciences, Flinders University, Adelaide, South Australia, Australia

${ }^{4}$ National Drug Research Institute, Curtin University, Perth, Western Australia, Australia

Acknowledgements We acknowledge the input of Professor Maree Teesson, Associate Professor Cath Chapman, Dr Katrina Champion, Dr Louise Birrell and Kate Ross in the design of this protocol as well as the members of the expert advisory group of the Positive Choices Indigenous project, of which this review is a part.

Contributors MS and LS conceptualised the study. NCN, JW and AES contributed to the conceptualisation of the study, including providing input in search terms. JW and AES contributed to definitions of levels of ecological model and cultural advice. All authors developed the study design and protocol. MS and BL wrote the first draft of the manuscript. LS, NCN, JW and AES read, revised and approved the final manuscript. MS is guarantor of the review.

Funding This work is supported by the Australian Government Department of Health. BL is supported by a National Health and Medical Research Council 
Postgraduate Scholarship (GNT1169377), NCN is supported by a National Health and Medical Research Council Career Development Fellowship (GNT1166377), and LS is supported by a National Health and Medical Research Council Translating Research into Practice fellowship (GNT1132853). The authors declare that the funders of the study have no influence in developing the protocol and for the conduct of the review.

Competing interests None declared.

Patient consent for publication Not required.

Provenance and peer review Not commissioned; externally peer reviewed.

Open access This is an open access article distributed in accordance with the Creative Commons Attribution Non Commercial (CC BY-NC 4.0) license, which permits others to distribute, remix, adapt, build upon this work non-commercially, and license their derivative works on different terms, provided the original work is properly cited, appropriate credit is given, any changes made indicated, and the use is non-commercial. See: http://creativecommons.org/licenses/by-nc/4.0/.

\section{REFERENCES}

1. Malaspinas AS, Westaway MC, Muller C, et al. A genomic history of aboriginal Australia. Nature 2016;538:207-14.

2. Nations U. State of the world's indigenous peoples. New York: UN Permanent Forum on Indigenous Issues (UNPFII), 2009.

3. Atkinson J. Trauma trails, recreating song lines: the transgenerational effects of trauma in Indigenous Australia. North Melbourne: North Melbourne: Spinifex Press, 2002

4. Osborne K, Baum F, Brown L. What works? A review of actions addressing the social and economic determinants of Indigenous health. Canberra / Melbourne: Australian Institute for Health and Welfare / Australian Institute for Family Studies, 2013

5. Anderson I, Robson B, Connolly M, et al. Indigenous and tribal peoples' health (The Lancet-Lowitja Institute Global Collaboration): a population study. Lancet 2016;388:131-57.

6. Australian Institute for Health and Welfare. The health and welfare of Australia's aboriginal and torres strait islander peoples: 2015. Canberra: AlHW, 2015.

7. Australian Health Ministers' Advisory Council. Aboriginal and torres strait islander health performance framework: 2014 report. Canberra: AHMAC, 2015.

8. Australian Institute for Health and Welfare. National drug strategy household survey detailed report. Canberra: Australian Government, 2013.

9. Gracey M, King M. Indigenous health part 1: determinants and disease patterns. Lancet 2009;374:65-75.

10. Dudgeon P, Calma T, Brideson T, et al. The Gayaa Dhuwi (Proud Spirit) Declaration - a call to action for aboriginal and torres strait islander leadership in the Australian mental health system. Advances in Mental Health 2016;14:126-39.

11. Australian Institute for Health and Welfare. Australian burden of disease study: impact and causes of illness and death in aboriginal and torres strait islander people 2011. Australian burden of disease study series. AlHW: Canberra, 2016.

12. Commonweath of Australia. National aboriginal and torres strait islander health plan 2013-2023. Commonwealth of Australia: Canberra, 2013.

13. Intergovernmental Committee on Drugs. National aboriginal and torres strait islander peoples' drug strategy 2014-2019. CanberraCommonwealth of Australia., 2014.

14. Altman JC, Biddle N, Hunter BH. A historical perspective on indigenous socioeconomic outcomes in Australia, 1971-2001. Aust Econ Hist Rev 2005;45:273-95.
15. Ring IT, Dixon T, Lovett RW, et al. Are Indigenous mortality gaps closing: how to tell, and when? Med J Aust 2016;205:11-12.

16. d'Abbs P. Widening the gap: The gulf between policy rhetoric and implementation reality in addressing alcohol problems among Indigenous Australians. Drug Alcohol Rev 2015;34:461-6.

17. Lovett R, Thurber KA, Maddox R. The Aboriginal and Torres Strait Islander smoking epidemic: what stage are we at, and what does it mean? Public Health Res Pract 2017;27:2741733.

18. Snijder M, Stapinski L, Lees B, et al. Substance Use Prevention Programs for Indigenous Adolescents in the United States of America, Canada, Australia and New Zealand: Protocol for a Systematic Review. JMIR Res Protoc 2018;7:e38.

19. Stone AL, Becker LG, Huber AM, et al. Review of risk and protective factors of substance use and problem use in emerging adulthood. Addict Behav 2012;37:747-75.

20. Burnette CE, Figley CR. Historical oppression, resilience, and transcendence: can a holistic framework help explain violence experienced by indigenous people? Soc Work 2017;62:37-44.

21. Midford R, Stockwell T, Gray D. Prevention of alcohol-related harm: community-based interventions, in national alcohol research agenda, commonwealth department of health and ageing. Canberra: Commonwealth Department of Health and Ageing, 2002:91-112.

22. Bronfenbrenner U, Morris PA. In: Damon W, Lerner R, The ecology of developmental processes, in handbook of child psychology. Hoboken, NJ US: John Wiley \& Sons, 1998:993-1028.

23. Hawkins JD, Catalano RF, Miller JY. Risk and protective factors for alcohol and other drug problems in adolescence and early adulthood: implications for substance abuse prevention. Psychol Bull 1992;112:64-105.

24. Bryden A, Roberts B, Petticrew M, et al. A systematic review of the influence of community level social factors on alcohol use. Health Place 2013;21:70-85.

25. Donovan JE. Adolescent alcohol initiation: a review of psychosocial risk factors. J Adolesc Health 2004;35:529.e7-18.

26. Ryan SM, Jorm AF, Lubman DI. Parenting factors associated with reduced adolescent alcohol use: a systematic review of longitudinal studies. Aust N Z J Psychiatry 2010;44:774-83.

27. Moher D, Liberati A, Tetzlaff J, et al. Preferred reporting items for systematic reviews and meta-analyses: the PRISMA statement. Ann Intern Med 2009;151:264-9.

28. Shamseer L, Moher D, Clarke M, et al. Preferred reporting items for systematic review and meta-analysis protocols (PRISMA-P) 2015: elaboration and explanation. BMJ 2015;349:97647.

29. Snijder M, Shakeshaft A, Wagemakers A, et al. A systematic review of studies evaluating Australian indigenous community development projects: the extent of community participation, their methodological quality and their outcomes. BMC Public Health 2015;15:1-16.

30. Snijder M, Stapinski L, Lees B, et al. Substance use prevention programs for indigenous adolescents in the United States of America, Canada, Australia and New Zealand: protocol for a systematic review. JMIR Res Protoc 2018;7:e38.

31. Fairley CK, Levy R, Rayner CR, et al. Randomized trial of an adherence programme for clients with HIV. Int J STD AIDS 2003;14:805-9.

32. Clifford A, Shakeshaft A. A bibliometric review of drug and alcohol research focused on Indigenous peoples of Australia, New Zealand, Canada and the United States. Drug Alcohol Rev 2017;36:509-22.

33. Munn Z, Moola S, Lisy K, et al. Methodological guidance for systematic reviews of observational epidemiological studies reporting prevalence and cumulative incidence data. Int J Evid Based Healthc 2015;13:147-53.

34. Long AF, Godfrey M, Randall T, et al. Developing evidence based social care policy and practice. Part 3: feasibility of undertaking systematic reviews in social care. Nuffield Institute for Health: Leeds, 2002. 